\title{
A College Student with Fulminant Hepatic Failure
}

\author{
Christie Crawford, $M D$
}

\section{Case Report}

A 19 year-old female college student presented to her pediatrician's office after experiencing three weeks of fatigue, abdominal pain, and nausea. At that time, the patient was diagnosed with a urinary tract infection and a viral illness and was given a prescription for Keflex. Over the next three days, the patient's symptoms worsened, and she developed weakness, shortness of breath, and anorexia. She also noticed yellowing of her skin and darkening of her urine, which prompted her to go to the emergency department at an outside hospital. At the outside hospital, many laboratory results were abnormal and her clinical condition worsened. She was transferred to the intensive care unit at Thomas Jefferson University Hospital (TJUH) for further evaluation and treatment.

The patient had no significant past medical, surgical history, or family history. She took a multivitamin and an oral contraceptive daily and denied the use of any herbal supplements or overthe-counter medications. She had no known drug allergies. She reported social alcohol and marijuana use, most recently one month ago, and denied tobacco use. She denied recent sexual activity or foreign travel. Review of systems was positive for shortness of breath, nausea, vomiting, abdominal pain, decreased urine output, dark urine, muscle cramps, headache, dizziness, jaundice, anorexia, and a six-pound weight gain over three weeks.

Upon admission, the patient's vital signs were within normal limits, and her physical exam was notable for jaundice, scleral icterus, diffuse abdominal tenderness and distension, ascites, and bilateral lower extremity pitting edema. Routine laboratories were notable for a white blood cell count of $51.9 \mathrm{~B} / \mathrm{L}$, a hemoglobin of $7.0 \mathrm{~g} / \mathrm{dL}$, and INR of 3.92 , and creatinine of $2.5 \mathrm{mg} / \mathrm{dL}$, a total bilirubin of $52.1 \mathrm{mg} / \mathrm{dL}$, a direct bilirubin of $21.8 \mathrm{mg} / \mathrm{dL}$, and alkaline phosphatase of less than 5 IU/L, a lactate dehydrogenase of $963 \mathrm{IU} / \mathrm{L}$, a haptoglobin of less than $6 \mathrm{mg} / \mathrm{dL}$, and a lactate of $28.4 \mathrm{mg} / \mathrm{dL}$.

\section{Hospital course}

With all clinical and laboratory findings pointing towards fulminant hepatic failure, a search for the cause was initiated. Tests for hepatitis B, hepatitis C, HIV, EBV, CMV, parvovirus, syphilis, SLE, autoimmune hepatitis, and acetomenopheninduced liver toxicity were all negative. The suspicion for Wilson Disease was high, but ceruloplasmin, urine and serum copper levels were still pending. Over the first two days of the patient's hospitalization, her condition worsened, and it became clear that she would require a liver transplant for survival. The patient was listed as status one for orthotopic liver transplantation with the presumptive diagnosis of Wilson Disease.

On the third day of hospitalization at TJUH, the patient began to get more confused and somnolent. The patient also became anuric with severe and progressive renal failure, requiring hemodialysis. The patient developed respiratory distress and was intubated. Additionally, she became hypotensive, and pressors were initiated. The patient was now severely acidemic, had a lactate of $197.7 \mathrm{mg} / \mathrm{dL}$, an INR of 6.77 with diffuse bleeding, and one of her pupils was fixed and dilated. The patient was taken to the operating room for hepatectomy, despite the fact that the donor liver transplant had not yet arrived at TJUH. Two hours later, the donor liver arrived and the patient underwent transplantation.

After the transplant was completed, the patient improved clinically, and her laboratory values also began to return towards normal. Her neurologic and cognitive function returned to baseline. She required one return trip to the operating room for bile duct reconstruction and was discharged from the hospital on post-operative day 19. The final diagnosis of Wilson Disease was made post-operatively when the patient's serum copper came back at $270 \mathrm{mcg} / \mathrm{dL}$ (normal range: $80-155$ ), and her urine copper came back at $2371.4 \mathrm{mcg} / \mathrm{dL}$ (normal range: $0.2-8$ ). Ceruloplasmin was within normal limits.

\section{Discussion}

Wilson Disease, also known as hepatolenitcular degeneration, is an autosomal recessive disorder caused by mutations in the ATP7B gene, which codes for a membrane-bound coppertransporting ATPase. When functioning properly, this ATPase transports copper into the bile so that it can be excreted from the body via the gastrointestinal tract. In patients with Wilson disease, copper cannot be excreted into the bile and thus accumulates in the liver bound to a protein called metallothionein. Mutations in the ATP7B gene also impair the incorporation of copper into ceruloplasmin. Excess copper in the liver causes increased generation of free radicals, which lead to liver damage. This progressive liver damage eventually leads to cirrhosis. A cirrhotic liver is unable to maintain the huge amount of accumulated copper, so the copper begins to leak into the bloodstream where it accumulates in and damages other tissues. ${ }^{1}$

Wilson Disease affects one in 30,000 live births. Symptoms rarely occur before age six and are almost always present by age thirty, with a mean age of onset of sixteen. Two times more females with Wilson Disease progress to fulminant hepatic failure than do their male counterparts. ${ }^{1,2}$

Neurologic symptoms, such as Parkinsonism, dystonia, cerebellar dysfunction, or pyramidal signs are the presenting features of Wilson Disease in $69 \%$ of patients. These neurologic manifestations result from accumulation of copper in the basal ganglia. Neurologic symptoms are often associated with psychiatric symptoms such as anxiety, depression, and pyschosis, but psychiatric symptoms are rarely seen in the absence of neurologic symptoms. Approximately $15 \%$ of patients with Wilson Disease 
present with signs and symptoms of cirrhosis, while another $5 \%$ have asymptomatic liver function test abnormalities. It is much less common for patients to present with isolated musculoskeletal complaints such as premature osteoporosis or osteoarthritis. It is also uncommon for patients to present with fulminant hepatic failure, as the patient in this case did. ${ }^{2}$

Kayser-Fleischer rings are brownish-gray rings seen around the iris that consist of copper deposits in Descemet's membrane that are commonly seen in Wilson Disease. They can only be appreciated on a slit lamp exam and are present in greater than $90 \%$ of Wilson Disease patients with neurologic and psychiatric symptoms. In patients with Wilson Disease without neurologic or psychiatric symptoms, Kayser-Fleischer rings will only be present $50 \%$ to $60 \%$ of the time. ${ }^{3}$

The patient in this case presented with fulminant hepatic failure which is unusual for Wilson Disease. Clinicians must have a high index of suspicion in order to make this diagnosis. Children and young adults who present with liver failure and also have a Coombs negative hemolytic anemia, a coagulopathy that is unresponsive to vitamin $\mathrm{K}$, renal failure, mild AST and ALT elevations, and/or alkaline phosphatase levels of less than $40 \mathrm{IU} / \mathrm{L}$ should be considered to have Wilson Disease until proven otherwise, as this presentation is associated with a very high mortality rate if not treated appropriately and in a timely fashion. ${ }^{4}$

The gold standard for diagnosing Wilson Disease is a liver biopsy with quantitative copper assay. However, this is rarely the first step in making the diagnosis. Patients with suspected Wilson Disease must first have liver function tests including coagulation studies, a complete blood count, a ceruloplasmin level, a 24-hour urine copper assay, and a thorough physical exam including a slit lamp exam. Patients with Kayser-Fleischer rings, a decreased serum ceruloplasmin, or urine copper levels greater than $40 \mathrm{mcg} /$ day need a liver biopsy to confirm the diagnosis. Copper levels of greater than $250 \mathrm{mcg} / \mathrm{g}$ on liver biopsy are consistent with a diagnosis of Wilson Disease. The work-up and diagnosis of Wilson Disease is a lengthy process and in patients with fulminant hepatic failure like the one in this case, treatment must be initiated before a diagnosis is finalized in order to give the patient the greatest chance of survival., ${ }^{3,4}$

Treatment of patients with Wilson Disease depends on whether or not the patient is symptomatic. Asymptomatic patients diagnosed via screening tests should be treated with the copper chelating agent penicillamine or with zinc, to remove copper that has already accumulated in the liver and prevent the further accumulation. For symptomatic patients, the copper chelating agent trientine should be used until laboratory tests confirm that copper levels have returned to the normal range, at which point patients may be switched to maintenance therapy with penicillamine or zinc. Patients with Wilson Disease must remain on copper chelation therapy for life, for if is stopped, copper will begin to reaccumulate. For patients that present with fulminant hepatic failure, emergent liver transplant is the only treatment available, and it is curative. ${ }^{4}$

Wilson Disease has an excellent prognosis if diagnosed and treated early. Neurologic and hepatic symptoms resolve with appropriate treatment. It is unclear as to whether there is an increased risk of hepatocellular carcinoma in patients with Wilson Disease. ${ }^{5}$ Prognosis for patients who present with fulminant hepatic failure is poor and depends on the availability of liver transplantation.

As Wilson Disease is a genetic disorder, screening of siblings and children of patients with the disease is necessary. Possible patients should be screened with slit-lamp examination, liver function tests, serum ceruloplasmin and copper levels, and 24hour urine copper excretion. If any of these tests are abnormal, a liver biopsy should be done to confirm the diagnosis. Occasionally, genetic testing may be performed, if the specific mutation of the ATP7B gene in the proband is known. When a patient's screening test for Wilson Disease is positive, treatment should be started immediately to ensure the best possible outcome.

\section{References}

1. Brewer GJ, Yuzbasiyan-Gurkan V. Wilson Disease. Medicine. 1992;71(3):139-64.

2. Taly AB, Meenakshi-Sundaram S, Sinha S, Swamy HS, Arunodaya GR. Wilson Disease: description of 282 patients evaluated over 3 decades. Medicine. 2007;86(2):112-21.

3. Steindl P, Ferenci P, Dienes HP, et. al. Wilson's Disease in patient presenting with liver disease - a diagnostic challenge. Gastroenterology. 1997;113(1):212-8.

4. Roberts RA, Schilsky ML. Diagnosis and treatment of Wilson Disease: an update. AASLD Practice Guidelines. Hepatology. 2008;47(6):2089-2111.

5. Walshe JM, Waldenstrom E, Sams V, Norlinder H, Westermark K. Abdominal malignancies in patients with Wilson's Disease. QJM. 2003;96:657-62. 